\title{
Caracterización clínica e histopatológica de niños con hepatitis autoinmune en un centro de referencia de Bogotá, Colombia
}

\section{Clinical and histopathological characterization of children with autoimmune hepatitis at a referral center in Bogotá, Colombia}

\author{
David Suárez¹, Rafael Andrade², José Fernando Vera³ , Rocío del Pilar López-Panqueva*
}

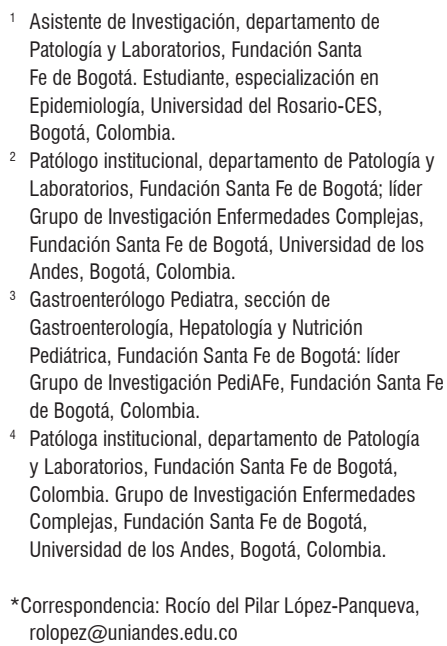

1 Asistente de Investigación, departamento de Patología y Laboratorios, Fundación Santa Fe de Bogotá. Estudiante, especialización en Epidemiología, Universidad del Rosario-CES, Bogotá, Colombia.

2 Patólogo institucional, departamento de Patología y Laboratorios, Fundación Santa Fe de Bogotá; líder Grupo de Investigación Enfermedades Complejas, Fundación Santa Fe de Bogotá, Universidad de los Andes, Bogotá, Colombia.

3 Gastroenterólogo Pediatra, sección de Gastroenterología, Hepatología y Nutrición Pediátrica, Fundación Santa Fe de Bogotá: líder Grupo de Investigación PediAFe, Fundación Santa Fe de Bogotá, Colombia.

4 Patóloga institucional, departamento de Patología y Laboratorios, Fundación Santa Fe de Bogotá, Colombia. Grupo de Investigación Enfermedades Complejas, Fundación Santa Fe de Bogotá, Universidad de los Andes, Bogotá, Colombia.

*Correspondencia: Rocío del Pilar López-Panqueva, rolopez@uniandes.edu.co

Fecha recibido: $29 / 01 / 18$

Fecha aceptado: 30/04/19

\begin{abstract}
Resumen
La hepatitis autoinmune (HAl) es una enfermedad hepática inflamatoria progresiva poco frecuente en niños y adolescentes, la cual es un reto diagnóstico para clínicos y patólogos. Describimos las características clínicas, bioquímicas e histopatológicas de 21 pacientes pediátricos con HAI diagnosticados en los últimos 14 años. Las biopsias hepáticas se reevaluaron para analizar detalladamente los hallazgos histopatológicos. De los 21 casos evaluados, $12(57,1 \%)$ fueron mujeres, la mediana de edad fue 14 años, y 17 (80,9\%) tenían HAI tipo 1. Los signos clínicos más frecuentes fueron ictericia $(66,7 \%)$ y coluria $(44,4 \%)$; también hubo evidencia de hipertensión portal con várices esofágicas $(47,1 \%)$ y esplenomegalia $(41,2 \%)$. El $11,8 \%$ de los pacientes tenía antecedentes de otras enfermedades autoinmunes. El $89,5 \%, 88,9 \%$ y $60,0 \%$ de los casos tenía elevación de aminotransferasas, hiperbilirrubinemia y bajos niveles de albúmina sérica, respectivamente. Las biopsias reevaluadas mostraron infiltrado linfoplasmocitario portal $(94,4 \%)$, hepatitis de interfase $(77,8 \%)$ y formación de rosetas $(50,0 \%)$. En el $42,9 \%$ de las biopsias se hallaron inclusiones hialinas en las células de Kupffer. Cerca del $33,5 \%$ de los casos mostró cirrosis en la biopsia inicial. A pesar del tratamiento inmunosupresor, 4 pacientes requirieron trasplante hepático y 2 están en lista de espera. La HAI en niños puede manifestarse con ictericia y coluria, signos de hipertensión portal, aminotransferasas elevadas, hiperbilirrubinemia y anticuerpos circulantes. Las inclusiones hialinas en las células de Kupffer pueden ser un hallazgo útil en el diagnóstico histopatológico de la HAl en niños.
\end{abstract}

Palabras clave

Hepatitis autoinmune, niño, biopsia, patología.

\begin{abstract}
Autoimmune hepatitis (AlH) is a progressive inflammatory liver disease. It is uncommon in children and adolescents, and is a diagnostic challenge for clinicians and pathologists. We describe the clinical, biochemical and histopathological characteristics of 21 pediatric patients with AlH diagnosed in the last 14 years. Liver biopsies were reassessed to analyze histopathological findings in detail. Of the 21 cases evaluated, 12 $(57.1 \%)$ were girls and young women, the median age was 14 years old, and $17(80.9 \%)$ had type $1 \mathrm{AlH}$. The most frequent clinical signs were jaundice $(66.7 \%)$, choluria $(44.4 \%)$, evidence of portal hypertension with esophageal varices (47.1\%), and splenomegaly (41.2\%). Histories of other autoimmune diseases were found in $11.8 \%$ of these patients. Elevated levels of aminotransferases were found in $89.5 \%$ of the patients, hyperbilirubinemia was found in $88.9 \%$, and $60.0 \%$ of the cases had low levels of serum albumin. Reassessed biopsies showed portal lymphoplasmocytic infiltrate $(94.4 \%)$, interface hepatitis $(77.8 \%)$ and rosette formation (50.0\%). Hyaline inclusions were found in Kupffer cells in $42.9 \%$ of the biopsies. About $33.5 \%$ of the cases showed cirrhosis at the initial biopsy. Despite immunosuppressive treatment, four patients required liver transplantation and two are on the waiting list. AlH in children can manifest with jaundice, choluria, signs of portal hypertension, elevated aminotransferases, hyperbilirubinemia and circulating antibodies. Hyaline inclusions in Kupffer cells may be a useful finding in the histopathological diagnosis of $\mathrm{AlH}$ in children.
\end{abstract}

\section{Keywords}

Autoimmune hepatitis, child, biopsy, pathology. 


\section{INTRODUCCIÓN}

La hepatitis autoinmune (HAI) es una enfermedad hepática inflamatoria progresiva, que se caracteriza por altos niveles de aminotransferasas e inmunoglobulina $G(\operatorname{Ig} G)$, anticuerpos circulantes y una respuesta favorable al tratamiento inmunosupresor (1-3). Aunque la HAI tiene un espectro amplio en su cuadro clínico, en niños y adolescentes a menudo se presenta de forma aguda y tiene un curso más agresivo que en pacientes de mediana edad y ancianos. Esta entidad puede progresar rápidamente a cirrosis e insuficiencia hepática si el diagnóstico es tardío y no se instaura un tratamiento oportuno (4).

Según la naturaleza de los anticuerpos séricos se han reconocido dos tipos de HAI: los anticuerpos antimúsculo liso (antiSMA) y los anticuerpos antinucleares (antiANA) definen la HAI tipo 1, mientras que los anticuerpos antimicrosomales hepatorrenales tipo 1 (antiLKM1) y los anticuerpos antiantígeno del citosol hepático tipo 1 (antiLC1) definen la HAI tipo 2 (5-7). Antes de la década de 1990, la epidemiología de la HAI en niños era imprecisa, ya que no existía un método estándar de diagnóstico; sin embargo, desde la creación del sistema de puntuación del Grupo Internacional de Hepatitis Autoinmunes, algunos países han estimado la incidencia y prevalencia de esta enfermedad (8). En un artículo publicado por Boberg se señaló que la incidencia de HAI tipo 1 entre poblaciones caucásicas (adultos y niños) de Europa y Norteamérica varía desde 0,1 a 1,9 casos por 100000 personas año, mientras que la prevalencia de HAI fue de 8,0 por 100000 habitantes en Islandia entre 1977 y 1979, y 16,9 por 100000 habitantes en Oslo, Noruega en 1995 (9). Adicionalmente, se ha descrito que la HAI tipo 1 representa dos tercios de los casos y habitualmente se presenta durante la pubertad, mientras que la HAI tipo 2 tiende a aparecer durante la infancia (10).

La evidencia histológica de daño inflamatorio en el hígado, compatible con el diagnóstico de HAI, es un prerrequisito para el diagnóstico de esta entidad $(8,11)$. La presencia de hepatitis de interfase con infiltrado linfoplasmocitario portal y periportal, formación de rosetas de hepatocitos y emperipolesis con linfocitos en el interior del citoplasma de los hepatocitos lesionados son los hallazgos característicos en la biopsia hepática (1-3). Recientemente, Tucker y colaboradores reportaron la presencia de gotas hialinas en el citoplasma de las células de Kupffer, que se tiñen con el ácido periódico de Schiff (PAS), como un nuevo hallazgo histológico para el diagnóstico de la HAI (12). Sin embargo, estos hallazgos no son patognomónicos y deben ser considerados en el contexto de los hallazgos clínicos, bioquímicos y serológicos de cada paciente (13).

El objetivo de este estudio es presentar las características clínicas y los hallazgos histopatológicos de pacientes pediátricos con HAI diagnosticados en nuestra institución durante los últimos 14 años.

\section{MATERIALES Y MÉTODOS}

Se realizó un estudio de corte transversal para identificar los pacientes menores de 18 años con diagnóstico de HAI en la Fundación Santa Fe de Bogotá, durante el período comprendido entre enero de 2004 y diciembre de 2017. El diagnóstico de HAI se estableció previamente según las normas descritas por el Grupo Internacional de Hepatitis Autoinmunes, publicadas en 1993 y revisadas en 1999 (14, 15). Se realizó la recolección retrospectiva de las siguientes variables clínicas y bioquímicas, usando el sistema de registro de historias clínicas disponible en nuestra institución: edad, sexo, forma de presentación clínica, manifestaciones clínicas, signos de hipertensión portal, historia familiar de enfermedades autoinmunes y asociación con enfermedades inmunológicas, pruebas de función hepática, niveles de bilirrubina, albúmina sérica, cociente normalizado internacional (INR), IgG, anticuerpos circulantes y necesidad de trasplante hepático. Asimismo, se reevaluaron las biopsias hepáticas disponibles en el departamento de Patología para analizar detalladamente los criterios histopatológicos, haciendo énfasis en el hallazgo descrito recientemente de gotas hialinas en las células de Kupffer (12).

Los datos se analizaron en el programa estadístico STATA ${ }^{\odot}$ (versión 12.0, StataCorp LP, College Station, Texas, EE. UU.). Las variables continuas se describieron con medidas de tendencia central y de dispersión según la distribución de los datos; y las variables cualitativas se describieron en términos de frecuencias absolutas y relativas. Este estudio fue revisado y avalado por el Comité Corporativo de Ética en Investigación de nuestra institución (número de referencia CCEI-2813-2015).

\section{RESULTADOS}

En el período de estudio se analizaron 280 biopsias hepáticas de pacientes pediátricos en el departamento de Patología; 22 de ellas con hallazgos histopatológicos y clínicos sugestivos o conclusivos de HAI. De los 22 pacientes evaluados, $12(57,1 \%)$ fueron mujeres y $9(42,9 \%)$ hombres, con una proporción mujer:hombre de 1,3:1. La mediana de edad fue 14 años y el rango de edad fue de 2 a 17 años. 17 niños $(80,9 \%)$ presentaron HAI tipo 1,1 $(4,8 \%)$ HAI tipo 2 y $3(14,3 \%)$ no tenían registro de anticuerpos. Los datos clínicos, los resultados de laboratorio y los hallazgos histopatológicos se resumen en la Tabla 1. Los síntomas más frecuentes fueron ictericia $(66,7 \%)$, coluria $(44,4 \%)$ y dolor abdominal $(27,8 \%)$, y los menos frecuentes fueron náuseas o vómito, cefalea o deterioro neuroló- 
Tabla 1. Hallazgos clínicos, bioquímicos e histopatológicos de niños con hepatitis autoinmune

\begin{tabular}{|c|c|c|c|}
\hline Modo de presentación $(n=18)$ & & Hallazgos de laboratorio+ & \\
\hline Hepatitis viral aguda (\%) & 72,2 & Bilirrubina total $(m g / d L)(n=18)$ & $3,0(0,5-22,6)$ \\
\hline Inicio insidioso (\%) & 22,2 & $\operatorname{AST}(U / L)(n=19)$ & $163,0(18,0-4448,0)$ \\
\hline Falla hepática aguda (\%) & 5,6 & $\operatorname{ALT}(U / L)(n=19)$ & $143,0(23,0-3939,0)$ \\
\hline Manifestaciones clínicas $(n=18)$ & & $\mathrm{FA}(\mathrm{U} / \mathrm{L})(\mathrm{n}=16)$ & $245,0(81,0-1390,0)$ \\
\hline Ictericia (\%) & 66,7 & Albúmina (g/dL) $(\mathrm{n}=15)$ & $3,6(1,1-4,9)$ \\
\hline Coluria (\%) & 44,4 & $\operatorname{INR}(n=14)$ & $1,2(0,9-1,9)$ \\
\hline Dolor abdominal (\%) & 27,8 & Gamma-glutamil transferasa $(U / L)(n=10)$ & $116,0(16,0-381,0)$ \\
\hline Acolia o fiebre (\%) & 22,2 & $\lg G(\mathrm{mg} / \mathrm{dL})(\mathrm{n}=8)$ & $2245,0(1041,0-3972,0)$ \\
\hline Anorexia (\%) & 16,7 & Hallazgos inmunológicos & \\
\hline Diarrea o distensión abdominal (\%) & 11,1 & $\operatorname{AntiSMA}(n=15)(\%)$ & 73,3 \\
\hline Amenorrea o prurito (\%) & 11,1 & $\operatorname{AntiANA}(n=18)(\%)$ & 66,7 \\
\hline Telangiectasias (\%) & 11,1 & Hallazgos patológicos $(n=18)$ & \\
\hline Náuseas o vómito (\%) & 5,6 & Infiltrado linfoplasmocitario (\%) & 94,4 \\
\hline Cefalea o deterioro neurológico (\%) & 5,6 & Hepatitis de interfase (\%) & 77,8 \\
\hline Epistaxis (\%) & 5,6 & Formación de rosetas (\%) & 50,0 \\
\hline Signos de hipertensión portal $(n=17)$ & & Gotas hialinas células de Kupffer (\%) & 42,9 \\
\hline Várices esofágicas (\%) & 47,1 & Cirrosis, probable o definitiva (\%) & 33,3 \\
\hline Esplenomegalia (\%) & 41,2 & Sin fibrosis (\%) & 22,2 \\
\hline
\end{tabular}

+Datos presentados como mediana (rango); ALT: alanina aminotransferasa; antiANA: anticuerpos antinucleares; antiSMA: anticuerpos antimúsculo liso; AST: aspartato aminotransferasa; FA: fosfatasa alcalina; IgG: inmunoglobulina G; INR: cociente normalizado internacional.

gico y epistaxis. En el 72,2\% de los casos, la presentación de los síntomas fue similar a la de una hepatitis aguda. En el examen físico, el 41,2\% (7/17) tenía evidencia de hipertensión portal con esplenomegalia y cerca de la mitad tenían várices esofágicas en la endoscopia.

En 2 pacientes $(11,8 \%)$, de 17 que tenían registros clínicos, se encontraron otras enfermedades autoinmunes. Un paciente tenía síndrome de activación macrofágica antes del diagnóstico de HAI. El otro caso fue diagnosticado con lupus eritematoso sistémico y glomerulonefritis membranoproliferativa. Solo un paciente $(5,9 \%)$ tenía antecedente familiar de enfermedad autoinmune. El 89,5\% de los casos presentó aumento de aminotransferasas y el $88,9 \%$ hiperbilirrubinemia. Los niveles de fosfatasa alcalina y el INR estaban elevados en el 33,3\% y 35,7\% de los casos, respectivamente. El 60,0\% de los niños tenía hipoalbuminemia y el $44,4 \%$ hipergammaglobulinemia.

El 85,7\% de los pacientes tenía biopsia hepática al inicio de la enfermedad (Figura 1). Las biopsias reevaluadas confirmaron el diagnóstico de HAI con base en la presencia de hepatitis de interfase $(77,8 \%)$, infiltrado linfoplasmocitario en los espacios porta $(94,4 \%)$ y formación de rosetas en las células hepáticas $(50,0 \%)$. Es preciso resaltar que en 6 de 18 niños (33,3\%), la biopsia hepática mostró cirrosis y, en la actualidad, a pesar del tratamiento con corticoides y azatioprina, 4 pacientes han sido sometidos a trasplante hepático y 2 están en lista de espera. Asimismo, la presencia de gotitas hialinas en el citoplasma de las células de Kupffer se comprobó en el 42,9\% de los casos.

\section{DISCUSIÓN}

La HAI es una causa rara de enfermedad hepática terminal en niños. Fallas en la regulación del sistema inmune, desencadenantes ambientales y susceptibilidad genética del huésped son mecanismos implicados en la patogénesis de esta enfermedad (8). Aunque la HAI fue descrita por primera vez en un grupo de mujeres jóvenes en 1950 por el profesor sueco Jan Waldenström, también puede afectar a los hombres ( $25 \%$ a $30 \%$ de todos los pacientes con HAI) y puede presentarse a cualquier edad y en todos los grupos étnicos $(2,5,16)$. De los 21 pacientes pediátricos con HAI descritos en el presente estudio, el $42,9 \%$ fue de sexo masculino y el $71,4 \%$ adolescentes ( $>12$ años). Además, cerca del 12\% de los casos tenía otras enfermedades autoinmunes asociadas y solo un paciente tenía antecedente familiar de enfermedad autoinmune. Esta cifra es ligeramente menor de la reportada en la literatura, donde cerca del $20 \%$ de los 

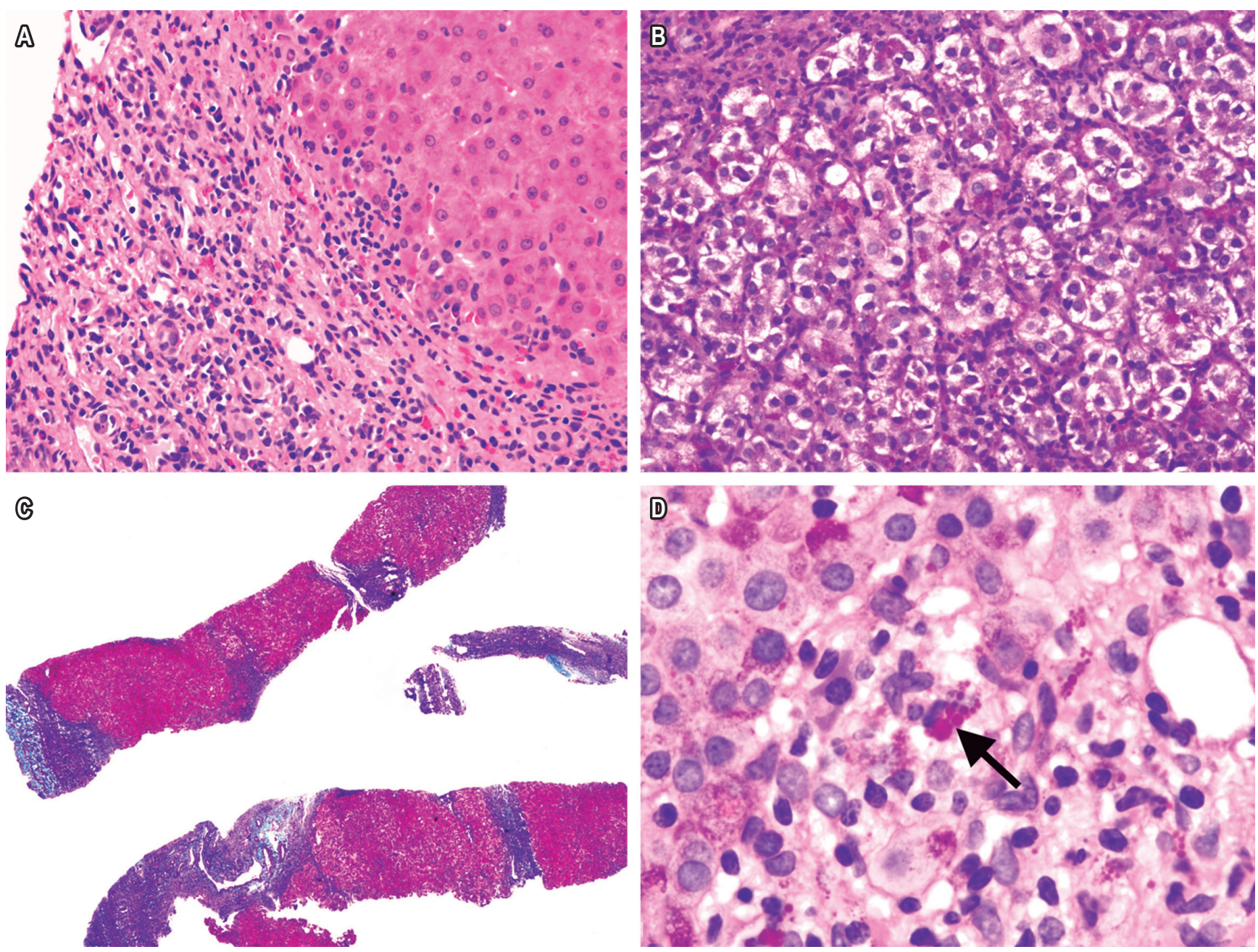

Figura 1. A y B. H\&E, biopsia hepática con HAI que evidencia hepatitis de interfase, infiltrado linfoplasmocitario portal (A 400x) y formación de rosetas (B 400x). C. Tricrómico de Masson, presencia de nódulos regenerativos rodeados por tabiques de fibrosis (40x). D. PAS-diastasa, gotas hialinas en las células de Kupffer (1000x).

niños con anticuerpos antiSMA/ANA y antiLKM1 positivos tienen enfermedades autoinmunes asociadas antes o después del diagnóstico de HAI, y el $40 \%$ de los casos tiene antecedente familiar de enfermedad autoinmune en familiares de primer grado (17). La razón para esta ligera discrepancia puede estar relacionada con las limitaciones en la recolección retrospectiva de los datos. $\mathrm{Al}$ ser un centro de referencia, algunas de las biopsias hepáticas que se procesan en el departamento de Patología son remisiones de otras instituciones y el acceso a la información clínica de estos pacientes puede ser limitado; sin embargo, se hizo todo lo posible para conseguir la información clínica de los casos incluidos en el estudio, consultando la fuente primaria, de ser posible, y los registros de historia clínica de la consulta externa de gastroenterología pediátrica.
Tres patrones de presentación clínica de la HAI en la edad pediátrica se han descrito en la literatura:

- En aproximadamente $40 \%$ de los casos, la presentación es similar a una hepatitis aguda con síntomas inespecífcos, tales como fatiga, náuseas, emesis, anorexia y dolor abdominal, seguido por ictericia, coluria y acolia

- En el $25 \%$ a $40 \%$ de los pacientes, el inicio es insidioso y se caracteriza por fatiga progresiva, ictericia recurrente, cefalea, anorexia, amenorrea y pérdida de peso, con una duración de varios meses e incluso años antes del diagnóstico

- Cerca del $10 \%$ de los casos pediátricos no tiene antecedente de ictericia y el diagnóstico de HAI se realiza debido a complicaciones de hipertensión portal, incluida esplenomegalia, hematemesis por várices esofágicas y diarrea crónica (18). 
En nuestra serie de pacientes, el 72,2\% de los casos debutó con un cuadro similar a una hepatitis aguda, el $22,2 \%$ tuvo un cuadro insidioso, pero llama la atención que el 41,2\% presentó esplenomegalia y el $47,1 \%$ várices esofágicas como signos de hipertensión portal, lo que indica un diagnóstico tardío (19). Aunque raramente los pacientes con HAI pueden desarrollar insuficiencia hepática fulminante, uno de nuestros casos ingresó con daño hepático grave y el 33,3\% tenía hallazgos sugestivos de cirrosis en la biopsia hepática inicial $(5,16)$. Se ha descrito que entre los pacientes con HAI, los hispanos en los Estados Unidos tienen mayor prevalencia de cirrosis (55\%), seguido por americanos de raza blanca (30\%) y asiáticos (29\%) (20). Asimismo, los casos sudamericanos son comúnmente niños pequeños con inflamación hepática severa (21). Se asume que la predisposición genética, factores de riesgo y razones socioeconómicas, tales como limitaciones en el acceso a los servicios de salud y el retraso en el diagnóstico, pueden explicar estas diferencias (2).

El 4,8\% de los casos fue diagnosticado con HAI tipo 2 y solo uno de nuestros pacientes tuvo anticuerpos negativos al momento del diagnóstico inicial; no obstante, los títulos de los anticuerpos pueden variar durante el curso de la enfermedad y los individuos seronegativos al momento del diagnóstico pueden expresar luego los anticuerpos (2). En los adultos, los títulos se consideran significativos cuando presentan una dilución $\geq 1: 40$ por inmunofluorescencia indirecta, mientras que en los niños, diluciones $\geq 1: 20$ para ANA y SMA o $\geq 1: 10$ para antiLKM1 soportan el diagnóstico de la HAI cuando se usan en combinación con otros hallazgos clínicos y de laboratorio sugestivos de la enfermedad (2-4). Además, los anticuerpos se correlacionan con la actividad de la enfermedad en pacientes pediátricos con HAI y pueden ser usados para monitorizar la respuesta al tratamiento (22). El 89,5\% de los pacientes presentó aumento de aminotransferasas, 88,9\% hiperbilirrubinemia, $60,0 \%$ hipoalbuminemia y $35,7 \%$ coagulopatía, estos dos últimos signos asociados con cirrosis y diagnóstico tardío. Debe tenerse en cuenta que hasta el $25 \%$ de los pacientes, especialmente niños, ancianos y casos con presentación aguda, tienen niveles de IgG dentro de los límites normales (11).

Debido a que las manifestaciones clínicas son tan variables y la presencia de anticuerpos séricos y los niveles elevados de IgG son muy inespecíficos, los hallazgos de la biopsia hepática son esenciales para confirmar el diagnóstico de la HAI y evaluar la severidad del daño hepático $(5,8)$. La hepatitis de interfase es un hallazgo típico, pero no exclusivo de la HAI y se caracteriza por la infiltración linfocitaria o linfoplasmocitaria portal y periportal con edema de los hepatocitos. En la HAI que se presenta de forma aguda o durante los episodios de recaída, a menudo se presenta hepatitis panlobular con necrosis en puente y, en el caso de presentación fulminante, necrosis masiva. Otros hallazgos descritos incluyen la rege- neración hepática con formación de rosetas y la emperipolesis $(2,5,8)$. En el 2008, el Grupo Internacional de Hepatitis Autoinmunes desarrolló un sistema con criterios simplificados para el diagnóstico de la HAI. La hepatitis de interfase con el infiltrado linfoplasmocitario, las rosetas y la emperipolesis son necesarios para categorizar un caso como típico; estos tres hallazgos deben estar presentes para obtener una puntuación histológica de 2 puntos $(23,24)$.

Algunos estudios han señalado que los criterios simplificados para el diagnóstico de la HAI tienen una excelente sensibilidad y especificidad en pacientes pediátricos, excepto en aquellos casos con falla hepática fulminante (25). Un hallazgo histológico novedoso descrito recientemente por Tucker y colaboradores es la presencia de gotas hialinas en el citoplasma de las células de Kupffer, que proporcionan información para distinguir la HAI de otras formas de hepatitis crónica (12). En nuestros casos, los hallazgos histopatológicos más frecuentes fueron el infiltrado linfoplasmocitario portal y la presencia de hepatitis de interfase. Las gotas hialinas estuvieron presentes en el 42,9\% de las biopsias hepáticas.

La severidad y presencia de ciertos cambios histológicos sirven como marcador para los diferentes fenotipos de la enfermedad (1). Un estudio realizado por Miao y colaboradores demostró que la emperipolesis está asociada con los cambios necroinflamatorios y fibróticos más severos en la HAI (26). Cabe destacar que hasta $24 \%$ de los pacientes puede presentar cambios en los conductos biliares, incluida la colangitis linfocítica, pleomórfica y destructiva. Estos casos deben ser investigados para una posible asociación con colangitis esclerosante primaria o colangitis/cirrosis biliar primaria (síndromes de sobreposición con HAI) (1). La biopsia inicial proporciona información sobre la actividad inflamatoria y el estadio de la fibrosis, lo cual ayuda a guiar las decisiones de tratamiento. Igualmente, las biopsias de seguimiento pueden ser necesarias para evaluar la respuesta al tratamiento, ya que la actividad inflamatoria residual predice la presencia de recaídas después de interrumpir la inmunosupresión (3). Por último, la biopsia permite la diferenciación entre la HAI y otras enfermedades hepáticas autoinmunes, tales como la cirrosis biliar primaria, la colangitis esclerosante primaria y la colangitis autoinmune (6).

En conclusión, la HAI se debe considerar en cualquier paciente pediátrico o adolescente con compromiso hepático agudo o crónico, en especial, si presenta elevación de aminotransferasas, hiperbilirrubinemia, hallazgos sugestivos de hipertensión portal y de otras enfermedades autoinmunes, asociados con la presencia de anticuerpos séricos e hipergammaglobulinemia. La presencia de signos de hipertensión portal, cirrosis, hipoalbuminemia o coagulopatía indican un diagnóstico clínico tardío. La biopsia hepática es importante tanto en el diagnóstico inicial como en el seguimiento a largo 
plazo de los pacientes con HAI. La presencia de gotas hialinas en el citoplasma de las células de Kupffer es un hallazgo histológico útil en el diagnóstico de la HAI en niños.

Como mencionamos previamente, dentro de las limitaciones de este estudio encontramos un número relativamente pequeño de pacientes pediátricos y adolescentes con HAI y las relacionadas con la recolección retrospectiva de los datos; sin embargo, los resultados obtenidos fueron equiparables a los descritos en la literatura.

\section{REFERENCIAS}

1. Wang Q, Yang F, Miao Q, Krawitt E, Gershwin M, Ma X. The clinical phenotypes of autoimmune hepatitis: a comprehensive review. J Autoimmun. 2016;66:98-107. doi: https:// doi.org/10.1016/j.jaut.2015.10.006.

2. European Association for the Study of the Liver. EASL Clinical Practice Guidelines: autoimmune hepatitis. J Hepatol. 2015;63:971-1004. doi: https://doi. org/10.1016/j.jhep.2015.06.030.

3. Manns M, Lohse A, Vergani D. Autoimmune hepatitisupdate 2015. J Hepatol. 2015;62:S100-11. doi: https://doi. org/10.1016/j.jhep.2015.03.005.

4. Mieli-Vergani G, Vergani D. Autoimmune liver diseases in children-what is different from adulthood? Best Pract Res Clin Gastroenterol. 2011;25:783-95. doi: https://doi. org/10.1016/j.bpg.2011.10.007.

5. Liberal R, Grant C, Mieli-Vergani G, Vergani D. Autoimmune hepatitis: a comprehensive review.J Autoimmun. 2013;41:12639. doi: https://doi.org/10.1016/j.jaut.2012.11.002.

6. Heneghan M, Yeoman A, Verma S, Smith A, Longhi MS. Autoimmune hepatitis. Lancet. 2013;382:1433-44. doi: https://doi.org/10.1016/S0140-6736(12)62163-1.

7. Carbone M, NeubergerJ. Autoimmune liver disease, autoimmunity and liver transplantation. J Hepatol. 2014;60:21023. doi: https://doi.org/10.1016/j.jhep.2013.09.020.

8. Pathak S, Kamat D. Autoimmune hepatitis in children. Pediatr Ann. 2018;47:e81-6. doi: https://doi. org/10.3928/19382359-20180126-01.

9. Boberg K. Prevalence and epidemiology of autoimmune hepatitis. Clin Liver Dis. 2002;6:635-47. doi: https://doi. org/10.1016/S1089-3261(02)00021-1.

10. Vitfell-Pedersen J, Jørgensen M, Müller K, Heilmann C. Autoimmune hepatitis in children in Eastern Denmark. J Pediatr Gastroenterol Nutr. 2012;55:376-9. doi: https:// doi.org/10.1097/MPG.0b013e3182602b20.

11. Gatselis N, Zachou K, Koukoulis G, Dalekos G. Autoimmune hepatitis, one disease with many faces: etiopathogenetic, clinico-laboratory and histological characteristics. World J Gastroenterol. 2015;21:60-83. doi: https:// doi.org/10.3748/wjg.v21.i1.60.

12. Tucker S, Jonas M, Perez-Atayde A. Hyaline droplets in Kupffer cells: a novel diagnostic clue for autoimmune hepatitis. Am J Surg Pathol. 2015;39:772-8. doi: https://doi. org/10.1097/PAS.0000000000000395.
13. Sahebjam F, Vierling J. Autoimmune hepatitis. Front Med. 2015;9:187-219. doi: https://doi.org/10.1007/s11684015-0386-y.

14. Johnson P, McFarlane I. Meeting report: International Autoimmune Hepatitis Group. Hepatology. 1993;18:9981005. doi: https://doi.org/10.1002/hep.1840180435.

15. Álvarez F, Berg P, Bianchi F, Bianchi L, Burroughs A, Cancado E, et al. International Autoimmune Hepatitis Group Report: review of criteria for diagnosis of autoimmune hepatitis. J Hepatol. 1999;31:929-38. doi: https:// doi.org/10.1016/S0168-8278(99)80297-9.

16. Liberal R, Grant C, Longhi M, Mieli-Vergani G, Vergani D. Diagnostic criteria of autoimmune hepatitis. Autoimmun Rev. 2014;13:435-40. doi: https://doi.org/10.1016/j. autrev.2013.11.009.

17. Gregorio G, Portmann B, Reid F, Donaldson P, Doherty D, McCartney M, et al. Autoimmune hepatitis in childhood: a 20-year experience. Hepatology. 1997;25:541-7. doi: https://doi.org/10.1002/hep.510250308.

18. Floreani A, Liberal R, Vergani D, Mieli-Vergani G. Autoimmune hepatitis: contrasts and comparisons in children and adults-a comprehensive review. J Autoimmun. 2013;46:716. doi: https://doi.org/10.1016/j.jaut.2013.08.004.

19. KrawittE. Autoimmune hepatitis. NEnglJ Med.2006;354:5466. doi: https://doi.org/10.1056/NEJMra050408.

20. Wong R, Gish R, Frederick T, Bzowej N, Frenette C. The impact of race/ethnicity on the clinical epidemiology of autoimmunehepatitis.J Clin Gastroenterol.2012;46:155-61. doi: https://doi.org/10.1097/MCG.0b013e318228b781.

21. Czaja A. Autoimmune hepatitis in special patient populations. Best Pract Res Clin Gastroenterol. 2011;25:689-700. doi: https://doi.org/10.1016/j.bpg.2011.09.011.

22. Gregorio G, McFarlane B, Bracken P, Vergani D, MieliVergani G. Organ and non-organ specific autoantibody titres and IgG levels as markers of disease activity: a longitudinal study in childhood autoimmune liver disease. Autoimmunity. 2002;35:515-9. doi: https://doi.org/10.10 $80 / 0891693021000056721$.

23. de Boer Y, van Nieuwkerk C, Witte B, Mulder C, Bouma G, Bloemena E. Assessment of the histopathological key features in autoimmune hepatitis. Histopathology. 2015;66:35162. doi: https://doi.org/10.1111/his.12558.

24. Balitzer D, Shafizadeh N, Peters M, Ferrell L, Alshak N, Kakar S. Autoimmune hepatitis: review of histologic features included in the simplified criteria proposed by the international autoimmune hepatitis group and proposal for new histologic criteria. Mod Pathol. 2017;30:773-83. doi: https://doi.org/10.1038/modpathol.2016.267.

25. Mileti E, Rosenthal P, Peters M. Validation and modification of simplified diagnostic criteria for autoimmune hepatitis in children. Clin Gastroenterol Hepatol. 2012;10:417-21. doi: https://doi.org/10.1016/j.cgh.2011.11.030.

26. Miao Q, Bian Z, Tang R, Zhang H, Wang Q, Huang S, et al. Emperipolesis mediated by CD8 T cells is a characteristic histopathologic feature of autoimmune hepatitis. Clin Rev Allergy Immunol. 2015;48:226-35. doi: https://doi. org/10.1007/s12016-014-8432-0. 